\title{
Bone Marrow Derivation of Interstitial Cells of Cajal in Small Intestine Following Intestinal Injury
}

\author{
Dengqun Liu, Fengchao Wang, Zhongmin Zou, Shiwu Dong, Junping Wang, Xinze Ran, \\ Chunxue Li, Chunmeng Shi, and Yongping Su
}

State Key Laboratory of Trauma, Burn and Combined Injury, Institute of Combined Injury, College of Preventive Medicine,
Third Military Medical University, Chongqing, 400038, China

Correspondence should be addressed to Yongping Su, suyp2003@yahoo.com.cn

Received 1 September 2009; Revised 24 December 2009; Accepted 27 January 2010

Academic Editor: Ronnie Gallagher

Copyright (๑) 2010 Dengqun Liu et al. This is an open access article distributed under the Creative Commons Attribution License, which permits unrestricted use, distribution, and reproduction in any medium, provided the original work is properly cited.

\begin{abstract}
Interstitial cells of Cajal (ICCs) in gastrointestinal tract are specialized cells serving as pacemaker cells. The origin of ICCs is currently not fully characterized. In this work, we aimed to study whether bone marrow-derived cells (BMDCs) could contribute to the origin of ICCs in the muscular plexus of small intestine using GFP-C57BL/6 chimeric mice.Engraftment of BMDCs in the intestine was investigated for GFP expression. GFP positive bone marrow mononuclear cells reached a proportion of $95.65 \% \pm 3.72 \%$ at different times in chimerism. Donor-derived cells distributed widely in all the layers of the gastrointestinal tract. There were GFP positive BMDCs in the myenteric plexus, which resembled characteristics of ICCs, including myenteric location, c-Kit positive staining, and ramified morphology. Donor-derived ICCs in the myenteric plexus contributed to a percentage ranging $9.25 \% \pm 4.9 \%$ of all the ICCs in the myenteric plexus. In conclusion, here we described that donor-derived BMDCs might differentiate into gastrointestinal ICCs after radiation injury, which provided an alternative source for the origin of the ICCs in the muscular plexus of adult intestine. These results further identified the plasticity of BMDCs and indicated therapeutic implications of BMDCs for the gastrointestinal dysmotility caused by ICCs disorders.
\end{abstract}

\section{Introduction}

Interstitial cells of Cajal (ICCs) are the specialized cell population of gastrointestinal cells, which perform essential roles in regulating the motility of gastrointestinal tract. According to their location in the intestine, ICCs could be divided into four subgroups, including submucosal ICCs (ICCs-SM), myenteric ICCs (ICCs-MY), intramuscular ICCs (ICCs-IM), and deep muscular plexus ICCs (ICCs-DMP). ICCs-MY are the most important population of ICCs in gastrointestinal tract. ICCs express $\mathrm{c}-\mathrm{Kit}$, which is a kind of tyrosine kinase receptor and at present widely used as the surface marker of ICCs. The most obvious morphological characteristics of them are that they usually possess processes and form a network, which distinguish them from mast cells, the other major c-Kit positive cells in the myenteric plexus of GI tract. Therefore, it could be necessary to define the gastrointestinal ICCs as a group of cells that have both
c-Kit expression and reticular morphological features [1]. Based on the current understanding of these cells, ICCs in gastrointestinal tract mainly have four different functions. ICCs in the myenteric plexus are pacemaker cells, which generate characteristic slow waves spontaneously $[2,3]$. They mediate inputs from enteric nervous system to smooth muscles. ICCs in the muscularis propria (ICCs-IM) have been indicated to mediate neurotransmission, including both the excitatory and inhibitory neuronal signals $[4,5]$. They also perform as mechanosensors through sodium channel on their membrane [6]. In addition, they could set the smooth muscle membrane potential gradient [7]. Among all these functions, the most important one is that they are the pacemaker cells that generate characteristic slow waves and regulate gastrointestinal motility.

As described above, ICCs are so important in regulating the gastrointestinal functions. Anyhow, they are also sensitive to harmful factors. For example, pulsating electromagnetic 
field (PEMF) exposure would result in the loss of ICCs [8]. It has also been reported that in several motility disorders, such as achalasia, mechanical ileus, intestinal pseudoobstructions, or malformations, reduced number of ICCs or dysfunction of their reticular structure could be observed [ 9 , 10]. Recent studies indicate that intestinal surgical operation would cause functional and morphological alterations of ICCs in mice, including disrupted electrical rhythmicity, neural responses, and interstitial cell networks [10].

During the past decades, it has been considered that proliferation of ICCs could only be detected in embryonic and neonatal periods. Recently, some investigators have shown that there are proliferative ICCs in the myenteric plexus after ICCs damage. For example, Mei et al. reported that ICCs could regenerate and restore their normal distribution after disruption by intestinal transection and anastomosis [11]. These findings excite researchers to explore the origin of ICCs.

Although the existence of ICCs has been identified for several decades, the progenitors of ICCs are still mysterious. At the beginning, ICCs had been presumed to be the descendants of neural crest cells. Using chimeric model and developmental observation, it was shown that ICCs came from the mesenchyme of the gut, but not the neural crest. Common precursors yielded ICCs-MY and longitudinal muscular cells [12-14]. In the stomach of postnatal murine, a small population of c-Kit ${ }^{\text {low }} \mathrm{CD} 44^{+} \mathrm{CD} 34^{+} \mathrm{IGF}^{-} \mathrm{IR}^{+}$cells had been isolated and characterized. These cells resembled embryonic ICCs precursors. And they could give rise to stomach ICCs when they were stimulated by soluble stem cell factor (SCF) and produce typical slow wave electricity [15]. Therefore, the progenitors of ICCs might exist in the muscles of gastrointestinal tract. However, all these studies are carried out from the developmental aspect. There are few reports concerning the origin of ICCs in adulthood, and no other cell types have been reported to have the ability to differentiate into ICCs.

Adult stem cells have been shown to have a great potential of differentiation. Not all adult stem cells are lineage restricted as previously believed. Among them, bone marrow-derived cells (BMDCs) have the potential to differentiate into several nonhematopoietic parenchymal cells, including neurons, hepatocytes, cardiomyocytes, and epithelial cells. Recently, BMDCs have been shown to successfully accelerate burn wound healing and promote the recovery of ischemic heart failure [16]. Therefore, it is promising to exploit the wide differentiation potentiality of BMDCs for the therapeutic treatment of human diseases. So it is necessary to study whether multipotential stem cells in other tissues, such as BMDCs, could be enrolled and reside in GI muscles and participate in the ICCs regeneration. In our previous work, we had found that BMDCs could long term repopulate the recipient's gastrointestinal epithelium [17]. In the present study, we attempted to determine whether BMDCs could differentiate into ICCs and contribute to ICCs regeneration in gastrointestinal tract using a model of GFP hematopoietic chimera. We found that BMDCs could migrate into the myenteric plexus and differentiate into ICCs in vivo after transplantation.

\section{Materials and Methods}

2.1. Animals. GFP-transgenic C57BL/6 mice were used as transplantation donors and purchased from the Model Animal Research Center of Nanjing University (AAALAC accredited). The strain code was C57BL/6TgN(ActbEGFP)1Osb [18]. The expression level of EGFP in bone marrow mononuclear cells was confirmed by flow cytometry and laser confocal microscope analysis. The GFP positive rate of BMDCs was more than $95 \%$. Wild-type C57BL/6 mice were used as transplantation recipients in this study, which were $6 \sim 8$ weeks old and purchased from Experimental Animals Center of Third Military Medical University. All the animals were acclimated to standard laboratory conditions (under a 12:12h light-dark cycle) and kept in specific pathogen free (SPF) grade environment. All animal experimental operations were conducted in accordance with the Animal Care Guidelines of the Third Military Medical University.

\subsection{Bone Marrow Preparation. BMDCs of GFP transgenic} mice were prepared as described previously. Briefly, bone marrow was aseptically harvested from femurs and tibias of donor mice by flushing bone cavity with cold DMEM/F12 medium containing $10 \%$ fetal bovine serum (FBS) (Hyclone, Beijing, China) using a syringe containing $100 \mathrm{IU}$ heparin with a 20-gauge needle. Marrow samples were sufficiently pipetted, centrifuged, and then filtered through a $20 \mathrm{um}$ nylon mesh to expel dregs. Then red blood cells were splited by RBC Lysis Buffer $\left(8.26 \mathrm{~g}\right.$ ammonium chloride $\mathrm{NH}_{4} \mathrm{Cl}$, $1 \mathrm{~g}$ potassium bicarbonate $\mathrm{KHCO}_{3}$, and $0.037 \mathrm{~g}$ EDTA in 1 liter $\mathrm{dd}_{2} \mathrm{O}$ ). All the marrow cells were resuspended in DMEM/F12 without FBS. Cell viability was determined by trypan blue exclusion experiment. About more than 95\% harvested cells were viable. Then concentration of BMDCs was determined by Beckman Coulter Z series Counter (Beckman Coulter, Hialeah, FL). BMDCs were resuspended in DMEM/F12 at a concentration of $1 \times 10^{7}$ viable nucleated cells per $350 \mu \mathrm{L}$, a transplantation volume for each recipient.

2.3. Irradiation and Transplantation. Forty C57BL/6 mice were used as transplantation recipients in the experiment. They were housed in autoclaved cages and treated with antibiotics for 10 days before irradiation and 2 weeks after irradiation (with $280 \mathrm{mg}$ erythromycin and $320 \mathrm{mg}$ gentamicin sulfate per liter of deionized drinking water). All the recipient mice were exposed to $10 \mathrm{~Gy}$ whole body irradiation (WBI) using a Cobalt-60 source (Theratron-780 model, MDS Nordion, Ottawa, ON, Canada). Such an irradiation dose had been used in many other transplantation studies $[19,20]$ and would cause severe gastrointestinal symptoms, such as diarrhea, bloody stool. Within three hours after transplantation, ten female recipient mice received injection of the same volume of PBS via tail vein as transplantation control. The other thirty mice were transplanted via tail vein with $1 \times 10^{7}$ GFP bone marrow cells in a volume of $350 \mu \mathrm{L}$. 
2.4. Determination of Chimeric Model. In order to examine the repopulation effect, bone marrow of chimeric mice was harvested as mentioned above. After depletion of RBC, mononuclear cells were examined by confocal microscope and flow cytometry. Because positive cells expressed GFP intrinsically, they were able to be recognized by $488 \mathrm{~nm}$ fluorescence.

2.5. Tissue Preparations and Detection of Donor's BMDCs. Tissue sections or whole mount preparations of small intestine were used for subsequent experiments. Fresh intestinal samples were immediately immersed into liquid nitrogen or fixed with $4 \%$ paraformaldehyde (PFA) or $100 \%$ acetone. Paraffin embedded intestinal samples were cut into $4 \mathrm{um}$ sections. After dewaxing and rehydration, antigen retrieval was carried out in $96^{\circ} \mathrm{C}$ for $10 \mathrm{~min}$ using sodium citrate solution $(0.01 \mathrm{M}, \mathrm{pH} 6.0)$. Activity of endogenous peroxidase was removed by incubation with $3 \% \mathrm{H}_{2} \mathrm{O}_{2}$ for 15 minutes at room temperature, and nonspecific binding was blocked by $3 \%$ normal goat serum incubation for $30 \mathrm{~min}$ at room temperature. Donor's BMDCs were identified by rabbit anti-GFP monoclonal antibody (1:200, AG279, Beyotime, Hangzhou, China). Horseradish peroxidase- (HRP-) conjugated goat antirabbit secondary antibody was used to bind the GFP antibody. Positive signals were shown by $\mathrm{DAB}$ response. Sections were counterstained with hematoxylin after IHC reaction.

2.6. Detection of Donor Derived ICCs. ICCs were recognized by c-Kit (ACK2, eBioscience, San Diego, CA, USA), and macrophages were demonstrated using rat anti-F4/80 monoclonal antibody (Abcam, Cambridge, MA, USA). Fluorescent double staining was employed to detect coexpression of GFP and c-Kit. Briefly, frozen sections or whole mount preparations were fixed in cold acetone for $15 \mathrm{~min}$ at $4^{\circ} \mathrm{C}$. After this, the samples were rinsed in PBS for $10 \mathrm{~min}$. Before adding primary antibody, nonspecific labeling was blocked by incubation in PBS containing 10\% normal goat serum and $0.2 \%$ Triton $\mathrm{X}-100$ at room temperature for $30 \mathrm{~min}$. Then they were incubated overnight at $4^{\circ} \mathrm{C}$ with primary antibody against c-Kit (1:100) or F4/80 (1:100). After rinsing in PBS three times for sufficient washing, Cy3-conjugated goat antirat secondary antibody (1:200) was added. After $1 \mathrm{~h}$ incubation in the dark and PBS washing, GFP antibody (1:200) and FITC-conjugated goat antirabbit secondary antibody (1:200) were sequentially added and, respectively, incubated overnight at $4{ }^{\circ} \mathrm{C}$ and for $30 \mathrm{~min}$ at room temperature. After three times washing, cell nuclei were counterstained with $0.3 \mu \mathrm{M} \mathrm{4}$ ',6-diamidino2-phenylindole, dihydrochloride (DAPI) (Molecular Probes, Inc., Eugene, OR, USA) in PBS for 1 min, which was done at room temperature and in the dark. Digital images were captured using TCS SP5 confocal laser scanning microscope (Leica, Germany) with an excitation wavelength appropriate for Cy3 $(552 \mathrm{~nm})$ or FITC (488 nm).

2.7. Statistics. SPSS version 16.0 (SPSS Inc., Chicago, USA) was used to carry out all statistical analyses. Data were
TABLE 1: The average percentage of GFP positive marrow cells in recipient's bone marrow ( $n=3$ at each point).

\begin{tabular}{lc}
\hline Time after transplantation & GFP positive rate $(\%)$ \\
\hline 1 month & $91.15 \pm 2.08$ \\
2 months & $92.05 \pm 3.15$ \\
3 months & $97.95 \pm 2.04$ \\
4 months & $98.05 \pm 1.58$ \\
5 months & $98.98 \pm 0.84$ \\
\hline
\end{tabular}

expressed as means \pm SEM. Differences in the data were evaluated by one-way analysis of variance (ANOVA). $P<.05$ was taken as a statistically significant difference.

\section{Results}

3.1. Establishment of GFP Positive Chimera Model. Mice transplanted with PBS in the control group died of bone marrow failure and intestinal symptoms within $5 \sim 7$ days after irradiation. On the other hand, all the mice in transplantation group successfully underwent the first week after irradiation with a survival rate more than $93.3 \%$. The survival rate of these two groups had significant difference $(P<.05)$. Transplantation of BMDCs could efficiently prevent recipients dying from bone marrow failure and gastrointestinal symptoms caused by irradiation. Bone marrow of chimeric mice at second month after transplantation was examined. Mononuclear cells in bone marrow were almost completely replaced by GFP positive cells (Figure 1(a)). By flow cytometry analysis, a representative image of GFP positive cells in all the marrow mononuclear cells at 2.5 months was shown in Figure 1(b). Chimeric rates at different timepoints were given in Table 1 , and the average level was $95.65 \% \pm 3.72 \%$. The results indicated that the GFP positive chimeric mice model was successfully established.

\subsection{Engraftment of Donor's BMDCs in Recipient's Intestine.} Using immunohistochemistry (IHC), it was shown that GFP positive BMDCs would migrate into intestine after transplantation. BMDCs appeared in the submucosal lamina propria during the first week, and then the quantity of these cells increased. There were positive cells in the muscular layer of intestine. GFP positive cells could be observed in the epithelium layer. Large amount of GFP positive BMDCs fulfilled the mesenchymal region of intestine (Figure 2(a)). Between the longitudinal and circular muscle cells, there were GFP positive cells with the appearance of processes (Figure 2(b), red arrow). The specificity of GFP antibody was confirmed by blank control staining. No positive signals could be seen in the same section without GFP antibody (Figure 2(c)). The muscular layer had been recovered from the irradiation induced-damage. No significant changes could be observed through HE staining (Figure 2(d)).

3.3. Differentiation of BMDCs towards Macrophages in the Muscular Layer. In order to examine the definite phenotype 


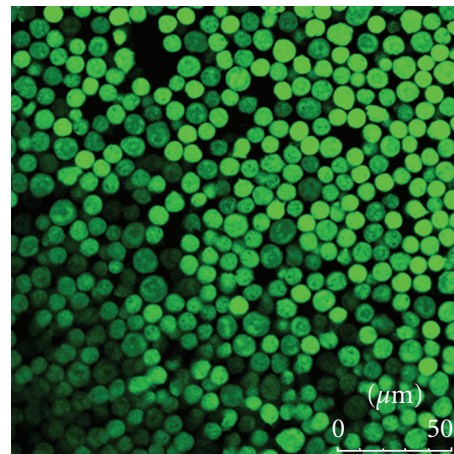

GFP

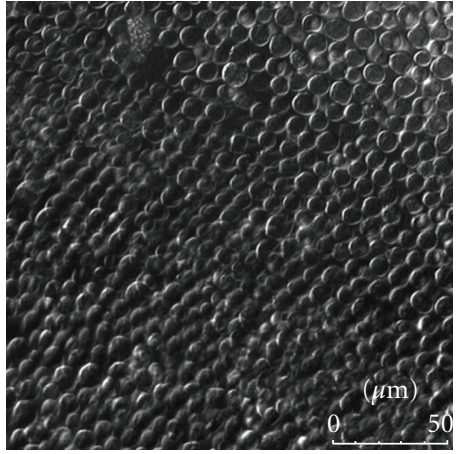

Bright field

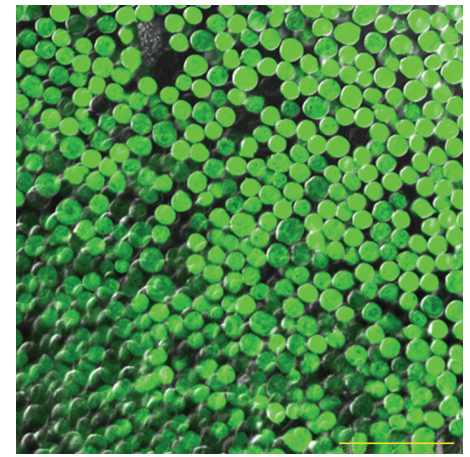

Overlay

(a)



(b)

FIGURE 1: Recipient's bone marrow was reconstituted by transplantation of donor's GFP positive BMDCs. (a) GFP positive marrow cells took up the majority of chimeric bone marrow two months after transplantation. Bar $=50 \mu \mathrm{m}$. (b) the percentage of GFP positive marrow cells in chimeric marrow at two months after transplantation was shown to be $92.05 \%$ by flow cytometry.

of GFP positive BMDCs in the muscular layer, macrophage differentiation was firstly observed. Using the antibody against F4/80, which was a widely-used surface marker for macrophages, it was shown that there were certain GFP positive macrophages. But the majority of positive macrophages were mainly found in the deep muscular plexus (DMP). For example, at the second month, both GFP positive and F4/80 positive cells could be detected in the DMP layer (Lower panel in Figure 3). GFP positive macrophages contributed to a proportion of $50.92 \pm 10.98 \%$ of all the macrophages in the DMP layer. BMDCs contributed to the generation of macrophages in DMP greatly.

3.4. Bone Marrow Derivation of ICCs-MY in Chimeric Intestine. As mentioned above, GFP positive cells in the DMP layer resembled macrophages appearance. We then tried to observe the phenotype of BMDCs in the myenteric plexus. In the myenteric plexus, GFP positive cells could also be detected. c-Kit positive ICCs-MY were well demonstrated using c-Kit antibody (ACK2). There were GFP and ACK2 double positive cells both in the intestinal sections and in the whole mount preparations. The representative images were demonstrated in Figures 4 and 5, in which the images were taken from recipient intestine 2.5 months after transplantation. Double positive cells were indicated by arrow and enlarged in the inset (Merged image in the lower panel of Figure 4). Donor-derived ICCs had bipolar appearance on the whole mount preparations (white arrow in merged image of the lower panel of Figure 5). Donorderived ICCs-MY reached a proportion of $9.25 \% \pm 4.9 \%$ among all the ICCs-MY in small intestine. These cells were distributed along the intestine without region specificity. Therefore, the transplanted BMDCs were able to engraft in smooth muscle of small intestine and differentiate into ICCs after the radiation injury.

\section{Discussion}

Adult stem cells exhibit more remarkable plasticity than previously believed, and BMDCs could contribute to several nonhematopoietic cell lineages. Both experimental and clinic studies have demonstrated that donor's BMDCs have multipotential differentiation ability in various organs of transplantation recipient, especially the nonhematopoietic differentiation capacity. They could differentiated into neurons, hepatocytes, and kidney tubular epithelial cells [21, 


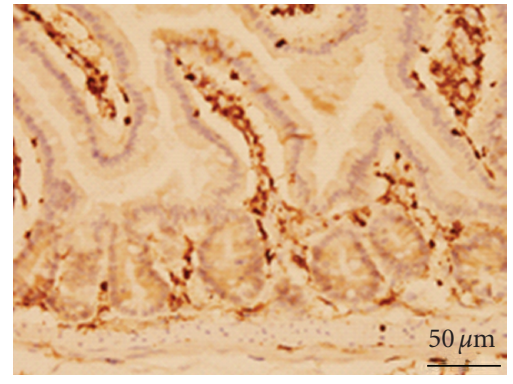

(a)

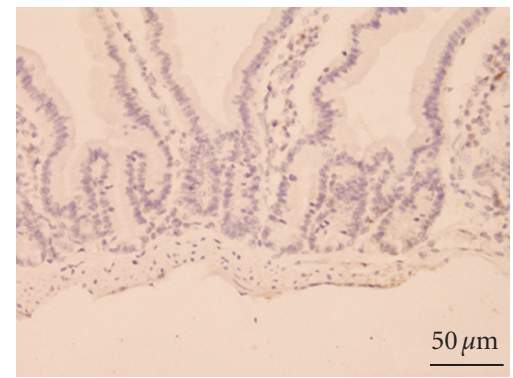

(c)

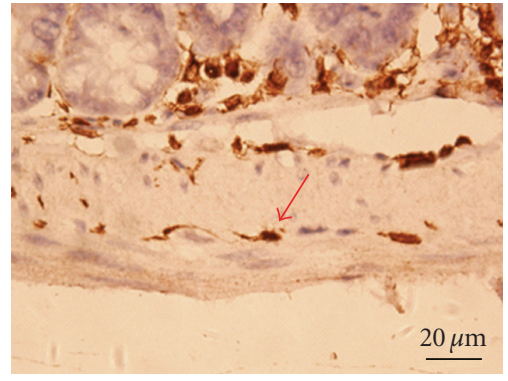

(b)

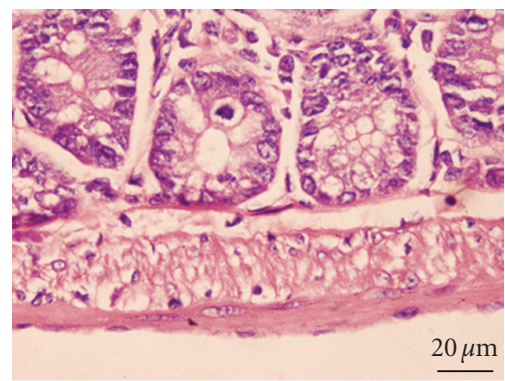

(d)

FIGURE 2: Detection of donor-derived BMDCs by GFP IHC in the small intestine 3 months after transplantation. (a) GFP positive BMDCs could be integrated into any part of intestine. (b) donor-derived cells could also be observed between longitudinal muscle and circular muscle, and there were some positive processes without ICCs body due to section reason (red arrow). (c) blank control without primary antibody on the serial section. No positive signals could be detected. (d) HE staining of small intestine from transplanted mice.

22]. Among all these findings, Okamato et al reported that BMDCs could also differentiate into epithelial cells to repopulate the damaged intestinal epithelium $[23,24]$. In the gastrointestinal tract, BMDCs have also been observed to differentiate into subepithelial myofibroblasts, which are important to regulate intestinal stem cell microenvironment [25]. Such exciting reports raised related research concerning the effects of BMDCs in regeneration of various injuries of gastrointestinal tract, such as dextran sulfate sodium (DSS) or trinitrobenzenesulfonic acid-(TNBS-) induced IBD model and alcohol-induced stomach epithelial injury [26-29]. All these studies had shown that BMDCs could participate in the injury restoration process in different damage models.

In our experiments, we established the chimera model using GFP transgenic mice. As confirmed by both flow cytometry and confocal scanning, the bone marrow of transplantation recipients had been repopulated by donor's BMDCs (Figure 1 and Table 1). In the previous study, we found that GFP positive cells could for long term exist in gastrointestinal tract. Because of ubiquitous GFP expression, staining of GFP allowed us to detect the engraftment and differentiation of donor-derived BMDCs conveniently. GFP positive BMDCs engrafted into each segment of the gastrointestinal tract without specificity. Besides epithelial engraftment, there were certain GFP positive cells in the tunica muscularis. For example, they could migrate into the myenteric plexus and deep muscular plexus as shown in Figure 2. Given the location of GFP positive cells, we tried to explore the possibility that whether BMDCs could differentiate into ICCs in gastrointestinal tract. Macrophages have also been shown to resident in the muscular layer [30]. Therefore, it was necessary for us to exclude whether these cells were macrophages. Through GFP and F4/80 double staining, it was demonstrated that GFP positive macrophages could be detected definitely. Anyhow, these cells were mainly found in the deep muscular plexus (Figure 3) and macrophages were negative for c-Kit. Using frozen sections and whole mount preparations, we found that there were GFP positive cells with several branches, which resembled the morphological characteristics of ICCs. In addition, GFP and c-Kit double positive cells could be observed in the myenteric plexus (Figures 4 and 5). The positive cells had at least two processes, which made them distinguishable from mast cells. Although mast cells in the myenteric plexus were also positive for cKit, these cells themselves were morphologically round and without processes. Besides this, they were rarely observed in the muscular layer of the mouse small intestine [31]. So the double positive cells were ICCs-MY, but not mast cells in the myenteric plexus. It encouraged us to believe that cKit positive BMDCs in the myenteric plexus were intestinal ICCs-MY according to the commonly accepted criteria, including cell location, surface marker, and morphological appearance. Therefore, the transplanted BMDCs were able to engraft in smooth muscle of intestine and differentiate into ICCs after the radiation injury. At present, it was difficult for us to carry out electrophysiological studies to study whether GFP positive cells could produce the slow wave electricity in this chimeric model, but a recent study had reported that bone marrow transplantation restored gut motility in Kit deficient mice [32], which indicated that these donor-derived 


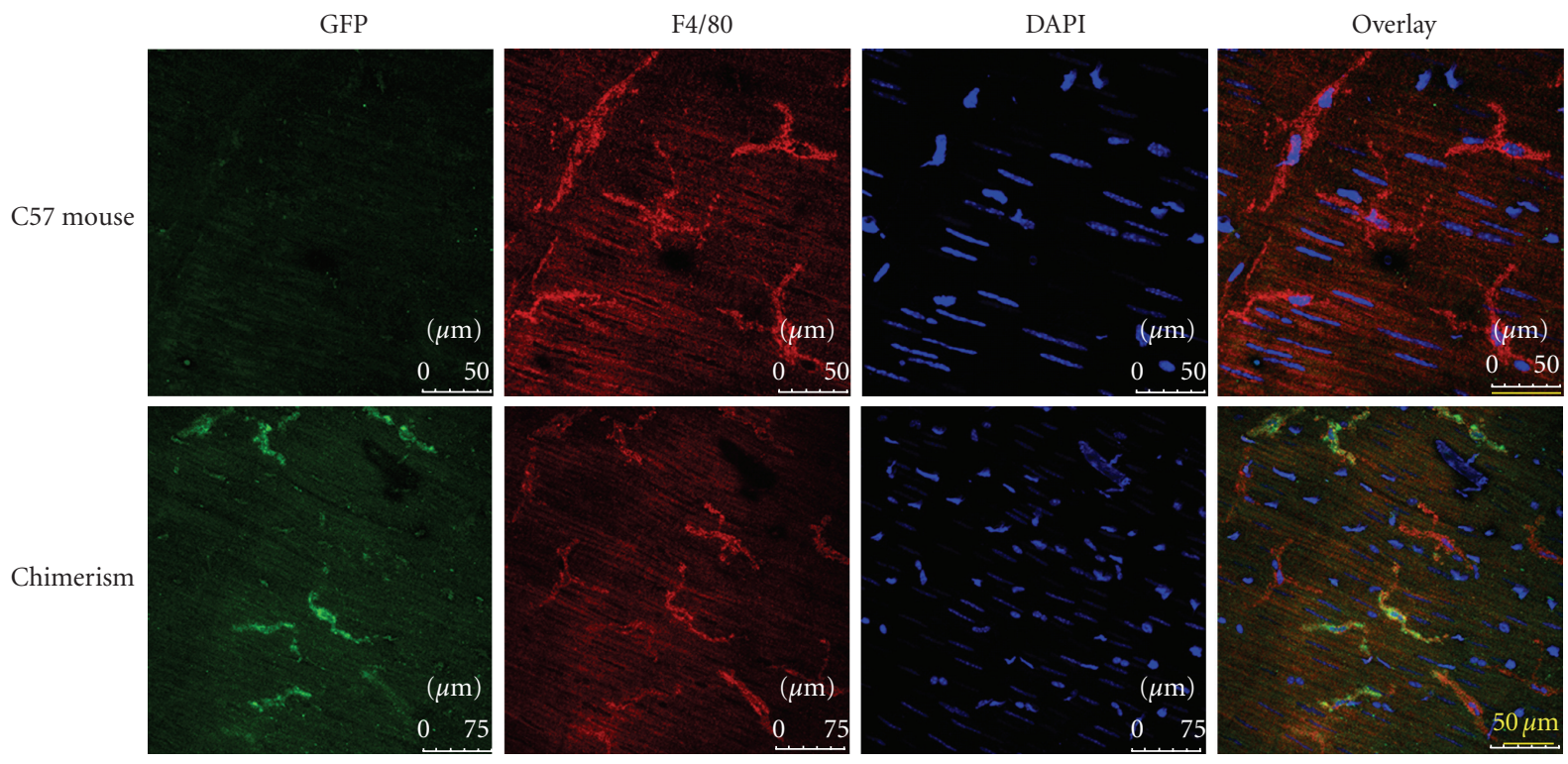

FIGURE 3: Whole mount preparation of small intestine from both normal C57 mouse and chimeric mouse. in this figure, donor's BMDCs were detected by GFP antibody. Macrophages were demonstrated by their specific marker F4/80. Upper panel: C57 mouse was used as normal control, in which all the macrophages were negative for GFP antibody. Bar $=50 \mu \mathrm{m}$. Lower panel: in the chimerism some macrophages were double positive for GFP and F4/80, suggesting that they were derived from the infused donor's BMDCs. $\mathrm{GFP}^{+} / \mathrm{F} / 80^{+}$cells were mainly found at the deep myenteric plexus and they were scattered in this layer, which distinguished them from ICCs. Bar $=50 \mu \mathrm{m}$.

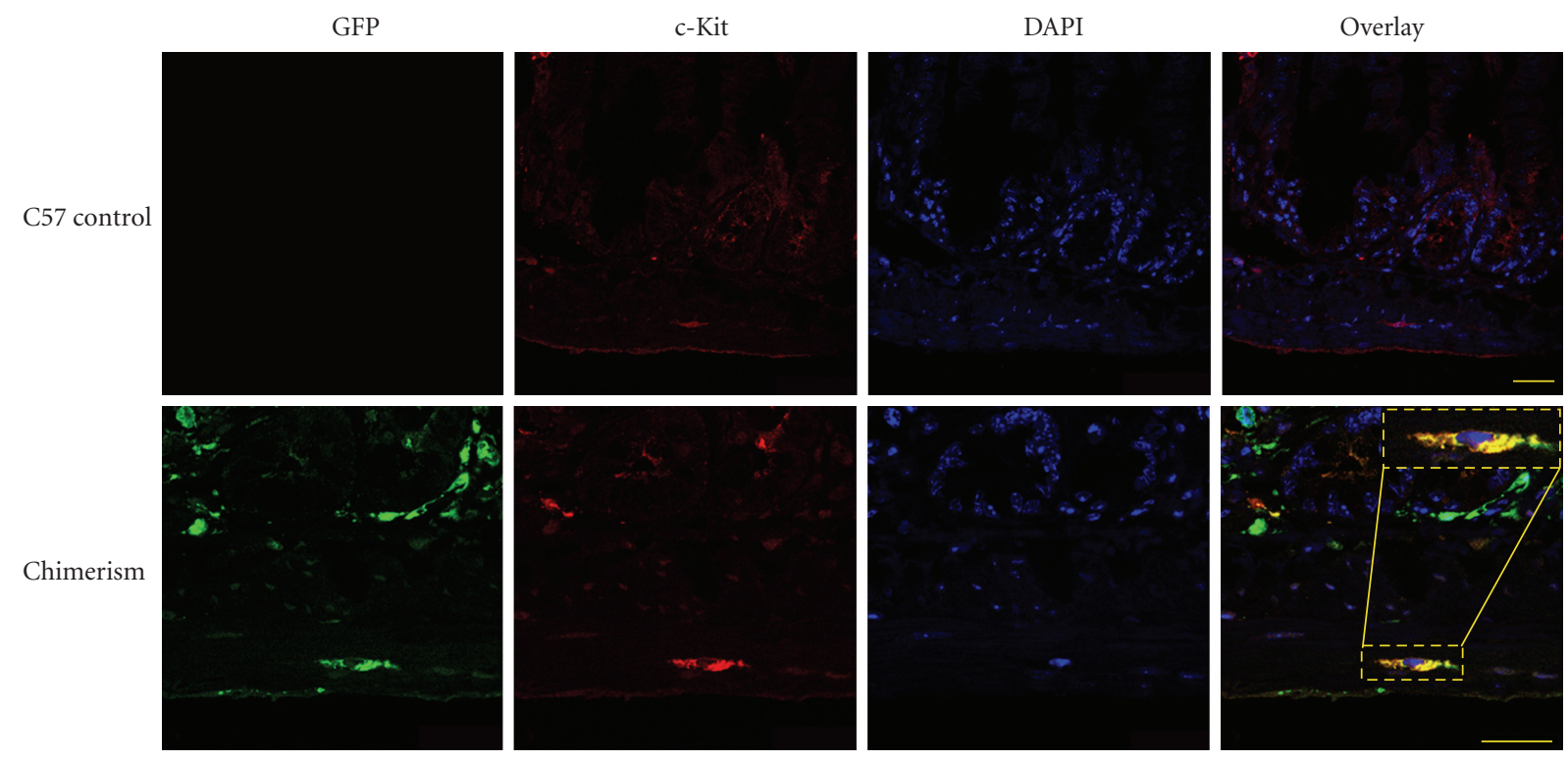

FIGURE 4: Confocal image of frozen section demonstrated donor derived ICCs-MY in small intestine of recipient mice 2.5 months after bone marrow transplantation. Upper panel: C57 mouse was used as negative control, in which there were no GFP signals in the intestine. Lower panel: In the chimerism, GFP and c-Kit double positive cells were shown in the merged image. Amplified image of interested cell was shown as inset. Bar $=25 \mu \mathrm{m}$.

ICCs played the same function as their wild-type mates. Liu Dengqun and Wang Fengchao contributed equally to this article.

Previous studies have demonstrated that ICCs originated from mesenchymal cells, but not the neural crest cells $[13,33]$. The proliferation of ICCs and extension of the processes are only observed in embryonic and neonatal periods in mammals, and even ICCs isolated from 6-day neonatal mice could not increase in number in vitro [34]. Recently, there were several investigations concerning the plasticity of ICCs and loss and recovery of ICCs had been reported $[35,36]$. Proliferative ICCs that could incorporate Brdu were observed after intestinal damage [37]. Another recent study has successfully identified a very small group 


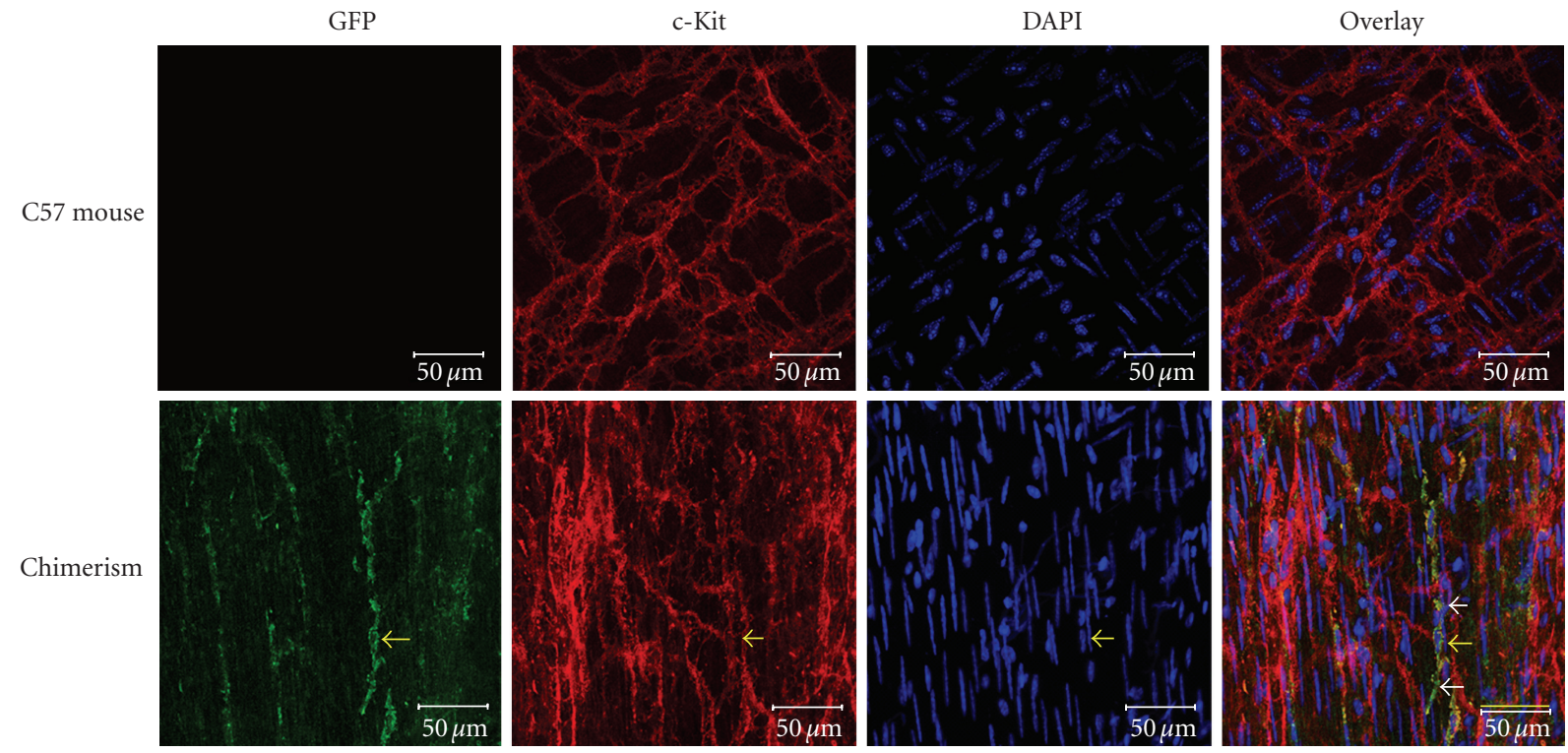

FIGURE 5: Representative immunofluorescent image of GFP and c-Kit double positive ICCs on whole mount preparation of small intestine. GFP protein was stained by FITC-labeled goat antirabbit IgG, and c-Kit was demonstrated by Cy3-conjugated goat anti-rat IgG. Upper panel: C57 mouse was used as negative control, in which there were no GFP signals in the intestine. Lower panel: GFP and c-Kit double positive cells were shown in chimeric intestine. Double positive cells could be found on the whole mount preparation, indicating that ICCs could be derived from BMDCs. Yellow arrow was indicated a double positive cell and white arrow directed to its two polar processes. Bar $=50 \mu \mathrm{m}$.

of $\mathrm{c}-\mathrm{Kit}^{\text {low }} \mathrm{CD} 44^{+} \mathrm{CD} 34^{+} \mathrm{IGF} \mathrm{IR}^{+}$cells in murine stomach as the putative progenitors of ICCs [15]. In the adult, the transdifferentiation of BMDCs has been well studied in many transplantation models. It has been identified that BMDCs are able to differentiate into adipocytes, osteocytes, chondrocytes. In the gastrointestinal tract, BMDCs have been reported to have the capacity to differentiate into epithelial cells $[17,38]$. In this study we used whole BMDCs as transplantation graft and identified that they could differentiate into ICCs. However, BMDCs used here were cell mixtures without purifying specific bone marrow stem cells (BMSCs). So we had not demonstrated that these ICCs were derived from bone marrow stem cells. It was possible that the GFP positive ICCs detected in the gut of recipients could be derived from surviving trapped donor cells. Given the current understanding of ICCs origin and characteristics of mesenchymal stem cells (MSCs), we presumed that MSCs, the most widely-investigated BMSCs, might be responsible for the donor-derived ICCs after transplantation.

Previous studies had demonstrated that transplanted BMDCs, such as MSCs, were preferentially recruited to the injured tissues caused by ischemia, trauma, and disease and could accelerate tissue regeneration. Herein, we confirmed the existence of transplanted bone marrow-derived ICCs in small intestine several months after irradiation and transplantation. It also meant that BMDCs as a source of ICCs under physiological condition could not be completely excluded. Because there were still GFP positive ICCs several months later after the radiation injury and transplantation. There were some other questions needed to be resolved in further study. One question was how BMDCs, especially BMSCs, such as MSCs, could differentiate into ICCs. Because only about $10 \%$ ICCs were donor-derived in the recipient's myenteric plexus, more efforts to study the mechanisms for BMDCs differentiation towards ICCs should be spent on induction of BMDCs (especially MSCs) in vitro. Another question was how to control the differentiation of BMDCs towards ICCs properly. It had been reported that abnormal ICCs might be the cause of gastrointestinal stromal tumors (GISTs). Transformation of MSCs had also been reported both in vivo and in vitro $[39,40]$. Therefore, when MSCs were used to repopulate ICCs damage, the safety was needed to be taken into account.

Both transdifferentiation and cell fusion have been used to interpret the formation of donor-derived nonhematopoietic cell phenotypes. Intestinal epithelial cells derived from donor's BMDCs were reported to be generated through cell fusion between BMDCs and intestinal stem cells [41]. We had also performed Y-FISH and GFP IHC staining to determine whether donor- derived ICCs were the products of cell fusion phenomenon, but in our models we did not find occurrence of cell fusion between donor's BMDCs and host's ICCs (unpublished data), suggesting that there was lack of evidence of cell fusion in the formation process of donor derived ICCs.

In conclusion, we showed that after irradiation and transplantation donor's BMDCs repopulated recipient hematopoiesis, migrated into gastrointestinal tract, and had the capacity to differentiate into ICCs in the myenteric plexus in the present study. This suggests a new origin of ICCs beyond gastrointestinal tract. All these results bring us a novel insight into the plasticity of BMDCs and an alternative cell resource for therapy of gastrointestinal dysmotility caused by ICCs disorders. In the future the results need to be 
confirmed using purified bone marrow stem cells to directly demonstrate the origin of donor cells residing in the gut. The specific formation mechanisms of donor-derived ICCs, whether cell fusion or transdifferentiation, might be further investigated in the future.

\section{Acknowledgments}

Dengqun Liu, Fengchao Wang and Chunmeng Shi contributed equally to this work. This work was supported by Basic Research Program (no. 2005CB522605), Innovation Group of Education Ministry (no. IRT0712), and CSTC2009BA5043. We appreciated Wei Sun and Li-ting Wang at Central laboratory, TMMU for their excellent technological assist in laser confocal scanning microscopy.

\section{References}

[1] G. Farrugia, "Interstitial cells of Cajal in health and disease," Neurogastroenterology and Motility, vol. 20, supplement 1, pp. 54-63, 2008.

[2] E. J. Dickens, G. D. S. Hirst, and T. Tomita, "Identification of rhythmically active cells in Guinea-pig stomach," Journal of Physiology, vol. 514, no. 2, pp. 515-531, 1999.

[3] H. M. Cousins, F. R. Edwards, H. Hickey, C. E. Hill, and G. D. S. Hirst, "Electrical coupling between the myenteric interstitial cells of Cajal and adjacent muscle layers in the Guinea-pig gastric antrum," Journal of Physiology, vol. 550, no. 3, pp. 829844, 2003.

[4] S. M. Ward, E. A. H. Beckett, X. Y. Wang, F. Baker, M. Khoyi, and K. M. Sanders, "Interstitial cells of Cajal mediate cholinergic neurotransmission from enteric motor neurons," Journal of Neuroscience, vol. 20, no. 4, pp. 1393-1403, 2000.

[5] H. Suzuki, S. M. Ward, Y. R. Bayguinov, F. R. Edwards, and G. D. S. Hirst, "Involvement of intramuscular interstitial cells in nitrergic inhibition in the mouse gastric antrum," Journal of Physiology, vol. 546, no. 3, pp. 751-763, 2003.

[6] P. R. Strege, Y. Ou, L. Sha, et al., "Sodium current in human intestinal interstitial cells of Cajal," American Journal of Physiology, vol. 285, no. 6, pp. G1111-G1121, 2003.

[7] G. Farrugia, S. Lei, X. Lin, et al., "A major role for carbon monoxide as an endogenous hyperpolarizing factor in the gastrointestinal tract," Proceedings of the National Academy of Sciences of the United States of America, vol. 100, no. 14, pp. 8567-8570, 2003.

[8] J. Kaszuba-Zwoińska, K. Gil, A. Ziomber, et al., "Loss of interstitial cells of Cajal after pulsating electromagnetic field (PEMF) in gastrointestinal tract of the rats," Journal of Physiology and Pharmacology, vol. 56, no. 3, pp. 421-432, 2005.

[9] C. J. Streutker, J. D. Huizinga, D. K. Driman, and R. H. Riddell, "Interstitial cells of Cajal in health and disease-part I: normal ICC structure and function with associated motility disorders," Histopathology, vol. 50, no. 2, pp. 176-189, 2007.

[10] H. Yanagida, H. Yanase, K. M. Sanders, and S. M. Ward, "Intestinal surgical resection disrupts electrical rhythmicity, neural responses, and interstitial cell networks," Gastroenterology, vol. 127, no. 6, pp. 1748-1759, 2004.

[11] F. Mei, B. Yu, H. Ma, H.-J. Zhang, and D.-S. Zhou, "Interstitial cells of Cajal could regenerate and restore their normal distribution after disrupted by intestinal transection and anastomosis in the adult Guinea pigs," Virchows Archiv, vol. 449, no. 3, pp. 348-357, 2006.
[12] H. M. Young, D. Ciampoli, B. R. Southwell, and D. F. Newgreen, "Origin of interstitial cells of Cajal in the mouse intestine," Developmental Biology, vol. 180, no. 1, pp. 97-107, 1996.

[13] L. Lecoin, G. Gabella, and N. Le Douarin, "Origin of the c-kitpositive interstitial cells in the avian bowel," Development, vol. 122, no. 3, pp. 725-733, 1996.

[14] S. Torihashi, S. M. Ward, and K. M. Sanders, "Development of c-kit-positive cells and the onset of electrical rhythmicity in murine small intestine," Gastroenterology, vol. 112, no. 1, pp. 144-155, 1997.

[15] A. Lorincz, D. Redelman, V. J. Horváth, M. R. Bardsley, H. Chen, and T. Ordög, "Progenitors of interstitial cells of Cajal in the postnatal murine stomach," Gastroenterology, vol. 134, no. 4, pp. 1083-1093, 2008.

[16] C. Yeo and A. Mathur, "Autologous bone marrow-derived stem cells for ischemic heart failure: REGENERATE-IHD trial," Regenerative Medicine, vol. 4, no. 1, pp. 119-127, 2009.

[17] L. Dengqun, W. Fengchao, Z. Zhongmin, et al., "Longterm repopulation effects of donor BMDCs on intestinal epithelium," Digestive Diseases and Sciences. In press.

[18] M. Okabe, M. Ikawa, K. Kominami, T. Nakanishi, and Y. Nishimune, "'Green mice' as a source of ubiquitous green cells,” FEBS Letters, vol. 407, no. 3, pp. 313-319, 1997.

[19] M. Komori, S. Tsuji, M. Tsujii, et al., "Involvement of bone marrow-derived cells in healing of experimental colitis in rats," Wound Repair and Regeneration, vol. 13, no. 1, pp. 109-118, 2005.

[20] A. R. Simard and S. Rivest, "Bone marrow stem cells have the ability to populate the entire central nervous system into fully differentiated parenchymal microglia," FASEB Journal, vol. 18, no. 9, pp. 998-1000, 2004.

[21] D. S. Krause, N. D. Theise, M. I. Collector, et al., "Multi-organ, multi-lineage engraftment by a single bone marrow-derived stem cell," Cell, vol. 105, no. 3, pp. 369-377, 2001.

[22] M. Alvarez-Dolado, R. Pardal, J. M. Garcia-Verdugo, et al., "Fusion of bone-marrow-derived cells with Purkinje neurons, cardiomyocytes and hepatocytes," Nature, vol. 425, no. 6961, pp. 968-973, 2003.

[23] R. Okamoto, T. Yajima, M. Yamazaki, et al., "Damaged epithelia regenerated by bone marrow-derived cells in the human gastrointestinal tract," Nature Medicine, vol. 8, no. 9, pp. 1011-1017, 2002.

[24] R. Okamoto, T. Matsumoto, and M. Watanabe, "Regeneration of the intestinal epithelia: regulation of bone marrow-derived epithelial cell differentiation towards secretory lineage cells," Human Cell, vol. 19, no. 2, pp. 71-75, 2006.

[25] M. Brittan, T. Hunt, R. Jeffery, et al., "Bone marrow derivation of pericryptal myofibroblasts in the mouse and human small intestine and colon," Gut, vol. 50, no. 6, pp. 752-757, 2002.

[26] Y. Kitamura, T. Okazaki, Y. Nagatsuka, Y. Hirabayashi, S. Kato, and K. Hayashi, "Immunohistochemical distribution of phosphatidylglucoside using anti-phosphatidylglucoside monoclonal antibody (DIM21)," Biochemical and Biophysical Research Communications, vol. 362, no. 2, pp. 252-255, 2007.

[27] R. Okamoto and M. Watanabe, "Cellular and molecular mechanisms of the epithelial repair in IBD," Digestive Diseases and Sciences, vol. 50, supplement 1, pp. S34-S38, 2005.

[28] T. Nishida, S. Tsuji, M. Tsujii, et al., "Cultured bone marrow cell local implantation accelerates healing of ulcers in mice," Journal of Gastroenterology, vol. 43, no. 2, pp. 124-135, 2008.

[29] M. Komori, S. Tsuji, M. Tsujii, et al., "Efficiency of bone marrow-derived cells in regeneration of the stomach after 
induction of ethanol-induced ulcers in rats," Journal of Gastroenterology, vol. 40, no. 6, pp. 591-599, 2005.

[30] H. Ozaki, T. Kawai, C. W. Shuttleworth, et al., "Isolation and characterization of resident macrophages from the smooth muscle layers of murine small intestine," Neurogastroenterology and Motility, vol. 16, no. 1, pp. 39-51, 2004.

[31] H. Maeda, A. Yamagata, S. Nishlkawa, et al., "Requirement of c-kit for development of intestinal pacemaker system," Development, vol. 116, no. 2, pp. 369-375, 1992.

[32] S. Ishii, S. Tsuji, M. Tsujii, et al., "Restoration of gut motility in Kit-deficient mice by bone marrow transplantation," Journal of Gastroenterology, vol. 44, no. 8, pp. 834-841, 2009.

[33] M. Klüppel, J. D. Huizinga, J. Malysz, and A. Bernstein, "Developmental origin and Kit-dependent development of the interstitial cells of Cajal in the mammalian small intestine," Developmental Dynamics, vol. 211, no. 1, pp. 60-71, 1998.

[34] M. Nakahara, K. Isozaki, J.-M. Vanderwinden, et al., "Dosedependent and time-limited proliferation of cultured murine interstitial cells of Cajal in response to stem cell factor," Life Sciences, vol. 70, no. 20, pp. 2367-2376, 2002.

[35] M.-S. Faussone-Pellegrini, M.-G. Vannucchi, O. Ledder, T.-Y. Huang, and M. Hanani, "Plasticity of interstitial cells of Cajal: a study of mouse colon," Cell and Tissue Research, vol. 325, no. 2, pp. 211-217, 2006.

[36] F. Mei, J. Han, Y. Huang, Z.-Y. Jiang, C.-J. Xiong, and D.-S. Zhou, "Plasticity of interstitial cells of Cajal: a study in the small intestine of adult Guinea pigs," Anatomical Record, vol. 292, no. 7, pp. 985-993, 2009.

[37] F. Mei, S. Guo, Y.-T. He, et al., "Apoptosis of interstitial cells of Cajal, smooth muscle cells, and enteric neurons induced by intestinal ischemia and reperfusion injury in adult Guinea pigs," Virchows Archiv, vol. 454, no. 4, pp. 401-409, 2009.

[38] J. Zhang, J.-F. Gong, W. Zhang, W.-M. Zhu, and J.-S. Li, "Effects of transplanted bone marrow mesenchymal stem cells on the irradiated intestine of mice," Journal of Biomedical Science, vol. 15, no. 5, pp. 585-594, 2008.

[39] A. Armesilla-Diaz, G. Elvira, and A. Silva, "p53 regulates the proliferation, differentiation and spontaneous transformation of mesenchymal stem cells," Experimental Cell Research, vol. 315, no. 20, pp. 3598-3610, 2009.

[40] M. Miura, Y. Miura, H. M. Padilla-Nash, et al., "Accumulated chromosomal instability in murine bone marrow mesenchymal stem cells leads to malignant transformation," Stem Cells, vol. 24, no. 4, pp. 1095-1103, 2006.

[41] A. Z. Rizvi, J. R. Swain, P. S. Davies, et al., "Bone marrowderived cells fuse with normal and transformed intestinal stem cells," Proceedings of the National Academy of Sciences of the United States of America, vol. 103, no. 16, pp. 6321-6325, 2006. 

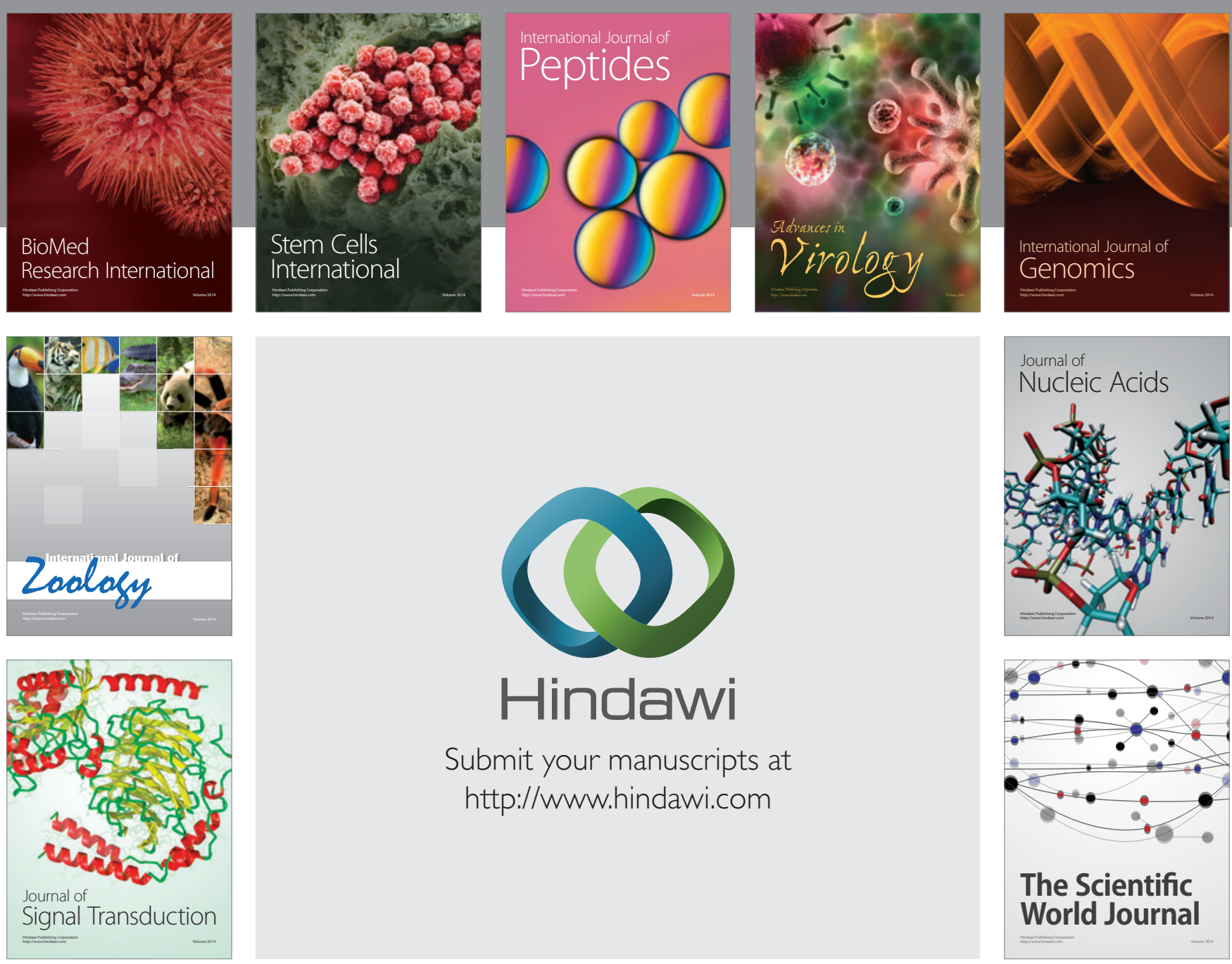

Submit your manuscripts at

http://www.hindawi.com
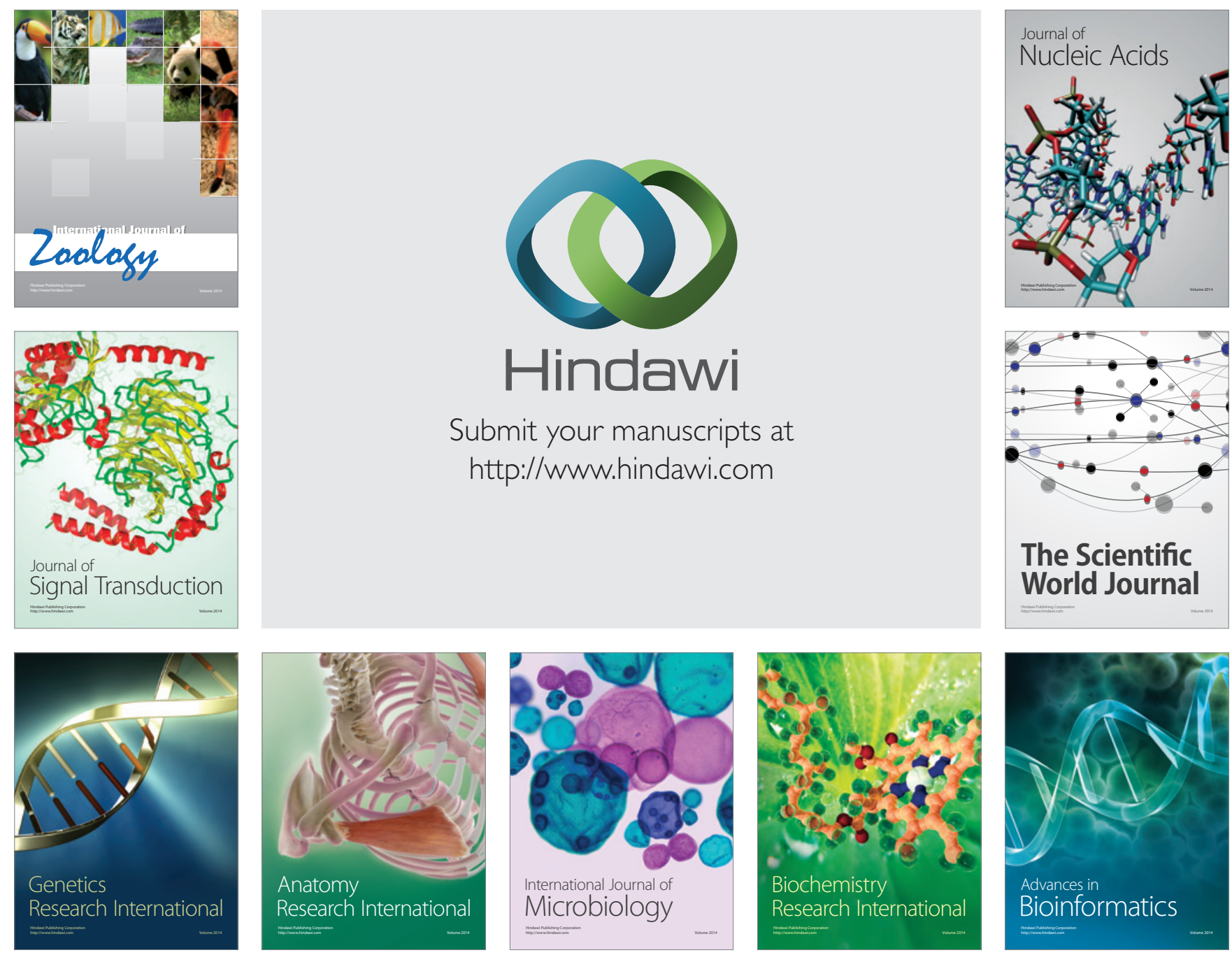

The Scientific World Journal
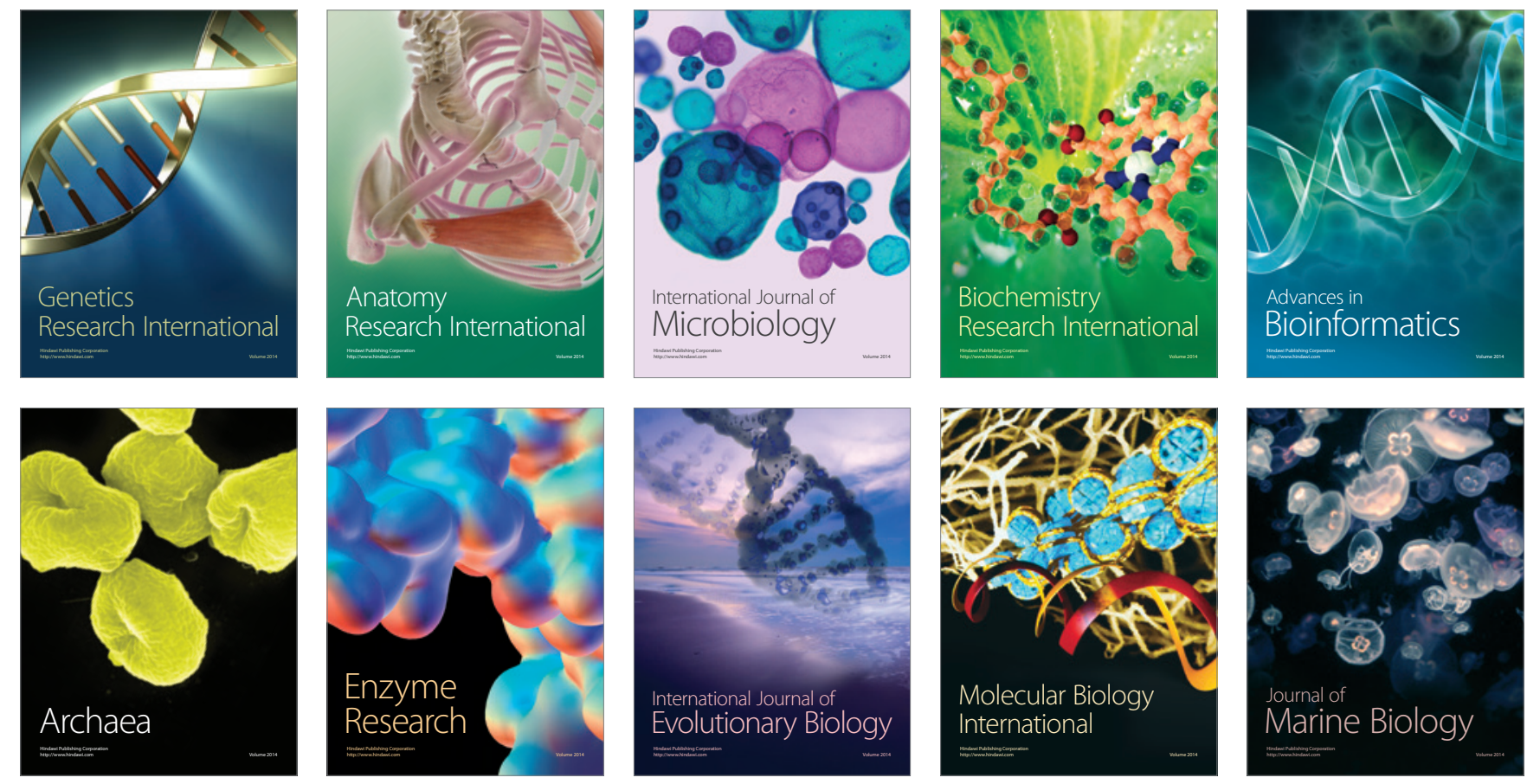